\title{
Estruturas recíprocas: revisão sistemática da literatura e identificação de pontos críticos para projeto e produção
}

\author{
Reciprocal frame structures: a systematic literature \\ review and identification of critical points for design and \\ production
}

\section{Caio Magalhães Castriotto \\ Gabriela Celani \\ Felipe Tavares da Silva}

\section{Resumo}

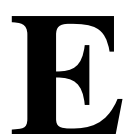

struturas recíprocas (ERs) são datadas do período neolítico, produzidas originalmente em madeira e compostas por elementos de dimensões reduzidas. Trata-se de um sistema estrutural que apresenta a capacidade de se adaptar a formas livres, complexas e com grande potencial estético, como indicado por diversas pesquisas contemporâneas. No entanto, acredita-se que essas estruturas poderiam fazer melhor uso das tecnologias digitais disponíveis de projeto, análises, fabricação e montagem. Este artigo tem o objetivo de identificar as atuais lacunas em que se pode concentrar esforços de pesquisa no sentido de tornar a tecnologia das ERs mais viável. Foi feita uma revisão sistemática da literatura (RSL) em sete bases de dados diferentes. Encontrou-se um total de 180 artigos, dos quais 49 foram selecionados para análise. Dentre eles, 27 identificaram lacunas existentes, que foram compiladas em cinco categorias: (a) dificuldades na concepção geométrica/estrutural; (b) necessidade de aprimoramento de ferramentas digitais; (c) dificuldade no projeto, análise, fabricação e montagem de conexões; (d) dificuldade de compreensão da real contribuição dos protótipos para aplicações em grande escala; e (e) necessidade de incorporar o processo de montagem no projeto de ERs. Foi possível concluir que o desenvolvimento de novos mecanismos de análise estrutural aliados à criação de novos detalhes construtivos pode contribuir para a expansão do uso das ERs.

Palavras-chave: Estruturas recíprocas. Nexorade. Revisão sistemática da literatura.
${ }^{1}$ Caio Magalhães Castriotto ${ }^{1}$ Universidade Estadual de Campinas Campinas - SP - Brasi

${ }^{2}$ Gabriela Celani ${ }^{2}$ Universidade Estadual de Campinas Campinas - SP - Brasil

${ }^{3}$ Felipe Tavares da Silva ${ }^{3}$ Universidade Federal da Paraíba João Pessoa - PB - Brasil

Recebido em 20/05/19 Aceito em 15/04/20

\begin{abstract}
Reciprocal Frame Structures (RFs) date from the Neolithic period, where they were originally produced in wood and comprised of small sized elements. It is also a structural system that can adapt to freeform and complex geometries, with great aesthetic potential, as indicated by several contemporary research studies. However, these structures could make better use of the digital technologies available for design, analysis, manufacturing and assembly. This paper aims to identify the current gaps where research efforts focus on in order to make $R F$ technology more viable. A systematic literature review (SLR) was performed in seven different databases. A total of 180 articles were found, of which 49 were selected for analysis. 27 of those papers identified existing gaps, which were then compiled into five categories: (a) difficulties in geometric / structural design; (b) need for enhancement of digital tools; (c) difficulty in connections design, analysis, manufacturing and assembly; (d) difficulty in understanding the real contribution of prototypes to large scale applications; and (e) need to incorporate the assembly process in the design of RF. The study concluded that the development of new structural analysis devices combined with the creation of new constructive details may contribute to expanding the use of RFs.
\end{abstract}

Keywords: Reciprocal frame structures. Nexorade. Literature systematic review. 


\section{Introdução}

Alguns sistemas construtivos muito antigos tinham o propósito de solucionar as limitações técnicas dos materiais e das tecnologias disponíveis. As reciprocal frames (estruturas recíprocas - ERs, tradução nossa), por exemplo, possibilitavam a construção rápida de abrigos e de coberturas com grandes vãos, com alguma complexidade geométrica. Historicamente, uma das vantagens da utilização desse sistema é o emprego de elementos lineares de pequena dimensão, originalmente em madeira, gerando uma composição estrutural em que cada elemento individual sustenta e é sustentado por outros elementos.

As ERs são datadas do período Neolítico, em cabanas primitivas (LARSEN, 2008) e também foram observadas durante o Império Romano, em uma ponte sobre o Rio Reno (LARSEN, 2014; PUGNALE; SASSONE, 2014). No século XII elas foram encontradas na arquitetura oriental, em coberturas conhecidas como "mandala roofs" e no século XIII surgiram as primeiras ERs na Europa, projetadas por Villard de Honnecourt. No início do século XVI Leonardo da Vinci desenvolveu esquemas para construções de pontes (Figura 1) com esse sistema estrutural, enquanto Sebastiano Serlio explicitou a possibilidade de se obter vãos maiores com a utilização de ERs (KOHLHAMMER; KOTNIK, 2011; PUGNALE; SASSONE, 2014).

Entre os séculos XVII e XVIII foram obtidos avanços significativos sobre os métodos de cálculo estrutural (como desdobramento do surgimento do cálculo diferencial). A partir da Revolução Industrial, no século XIX, foram desenvolvidos novos materiais estruturais, tais como o aço e o cimento Portland. Nesse mesmo período os produtos de madeira passaram por um processo de estandardização e produção em massa. Com isso, surge também um novo interesse pelas ERs, pela facilidade de montagem, com destaque para o desenvolvimento do sistema Zollinger, no início do século XX.

Diante da possibilidade de uso de ferramentas computacionais (paramétricas e algorítmicas) e equipamentos de controle numérico, surge novamente o interesse em explorar essas estruturas. Alguns autores têm demonstrado como é possível produzi-las com geometrias complexas em projetos personalizados. Essas inovações estão muitas vezes ligadas à possibilidade de solucionar problemas antigos graças aos avanços tecnológicos e científicos. Paralelamente, duas das principais características da arquitetura contemporânea são a sustentabilidade e a complexidade formal. Nesse contexto, as ERs em madeira ganham destaque, uma vez que além do potencial estético faz uso de um material de origem renovável, de baixo impacto ambiental e que pode ser beneficiado pelo uso de tecnologias digitais de projeto, fabricação e montagem.

Em suma, existem diversas possibilidades ainda inexploradas de aplicação das estruturas recíprocas. Por isso, faz-se necessária uma revisão sobre o tema. Com esse objetivo, este artigo apresenta uma revisão sistemática da literatura sobre estruturas recíprocas em madeira. Como resultado, espera-se identificar lacunas que permanecem e que podem, potencialmente, ser solucionadas com o uso de recursos digitais.

Figura 1 - Modelo da estrutura para a ponte de Leonardo da Vinci, um tipo de ER

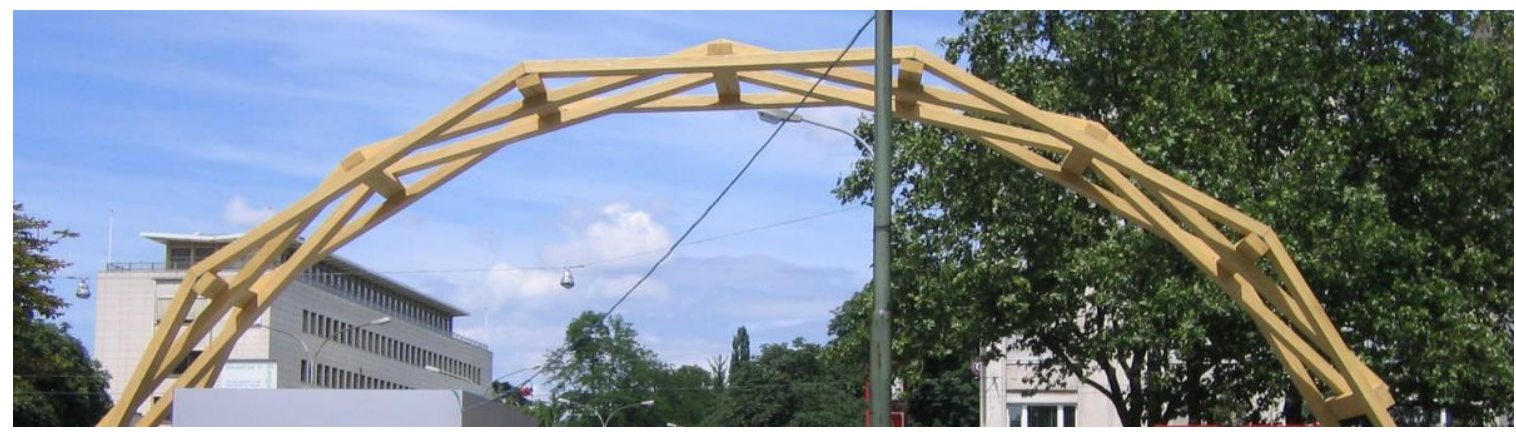

Fonte: Flominator (2020). 


\section{Método}

A utilização da revisão sistemática da literatura (RSL) é uma forma de identificar, avaliar, analisar e interpretar publicações de pesquisas relevantes que estejam relacionadas com determinado tópico, questão, fenômeno ou nicho. Os resultados encontrados permitem uma visão ampla e fundamentada sobre a área de análise, sendo um mecanismo de problematização e argumentação de pesquisa (DRESCH; LACERDA; ANTUNES JÚNIOR, 2015). Para a realização desta RSL, foram adotadas as seguintes etapas, sugeridas por Kitchenham (2004) e utilizadas por Machado e Ruschel (2018):

(a) planejamento: definição dos mecanismos e encadeamentos de busca;

(b) condução: aplicação de critérios de filtragem da busca;

(c) comunicação: quantificação e sintetização dos resultados obtidos.

\section{Planejamento}

O planejamento para a realização da RSL foi construído a partir de um encadeamento das seguintes questões (KITCHENHAM, 2004; MACHADO; RUSCHEL, 2018):

(a) Qual a relevância do tema?

(b) Quais são as questões de pesquisa?

(c) Qual o objetivo da RSL?

(d) Quais as estratégias de busca?

(e) Quais bases de dados seriam utilizadas?

(f) Quais os critérios de inclusão ou exclusão?

(g) Quais as estratégias para a extração de dados?

Pode-se dizer que a relevância do tema é encontrada na possibilidade de uso de tecnologias digitais de projeto, de fabricação e de montagem, dado o elevado grau de precisão e de personalização obtidos, sem precedentes na história. As estruturas recíprocas aparecem como solução construtiva em obras de distintos períodos da história, com características interessantes para a economia de material e a conformação de geometrias complexas. Porém, os avanços sobre esse tipo de sistema construtivo sempre foram difíceis e lentos, principalmente pela dificuldade de cálculos e do controle de qualidade dos materiais - algo que pode ser resolvido com maior precisão mais recentemente.

As ERs são um sistema com características únicas, com a possibilidade de criar uma extensa variedade de geometrias e com inúmeras maneiras de aplicação, mas que têm sido utilizadas apenas em ocasiões muito pontuais (LARSEN, 2008). Além disso, trata-se de um sistema adequado para o uso da madeira, que vem sendo reconhecida cada vez mais como um material sustentável por excelência, por se tratar de um recurso renovável e de baixo impacto ambiental (GAUZIN-MULLER, 2005). Ao mesmo tempo, as tecnologias recentemente desenvolvidas para o tratamento da madeira contra agentes biológicos e para a criação de produtos engenheirados, como a madeira laminada cruzada (CLT), fazem esse material ser muito mais seguro e controlado que no passado.

O objetivo da RSL foi identificar possíveis lacunas para o desenvolvimento de estruturas recíprocas, realizando a compilação e classificação de pesquisas e experiências recentes sobre o tema. Os objetivos específicos da RSL foram:

(a) definir e identificar as origens, tipos e características das estruturas recíprocas; e

(b) identificar o potencial de utilização de recursos digitais para solucionar uma determinada classe de problemas.

Com o intuito de aprofundar as discussões e promover uma visão mais global sobre o assunto, as estratégias de busca restringiram os resultados por artigos publicados em periódicos ou congressos científicos, disponíveis integralmente em meio eletrônico e que apresentassem relação entre ER e madeira ou ER e fabricação digital. Além disso, um levantamento inicial mostrou que o termo "reciprocal structure" é uma nomenclatura utilizada, predominantemente, na área da saúde e biologia, e que nas áreas de arquitetura e construção civil as ERs possuem diferentes nomes, como nexorade, lamella structure e Zollinger. Desse modo, os termos ou strings de busca utilizados foram: 
(a) "Nexorade";

(b) "Reciprocal Frame";

(c) "Reciprocal Frame" AND "Wood";

(d) "Reciprocal Frame" AND "Timber";

(e) "Reciprocal Frame" AND "Digital Fabrication";

(f) "Reciprocal Frame" AND "Lamella"; e

(g) "Reciprocal Frame” AND “Zollinger".

Foram utilizadas as seguintes bases de dados para a busca:

(a) Periódicos CAPES;

(b) Sage Journals;

(c) CumInCAD;

(d) Ingenta Connect;

(e) Compendex;

(f) SCOPUS; e

(g) Web of Science.

Para refinar os resultados obtidos, foram definidos critérios de inclusão e exclusão de artigos. Foram incluídos todos os trabalhos que descrevem uma experiência prática de concepção, fabricação e montagem de uma ER e/ou que discutem do ponto de vista teórico os avanços necessários para o desenvolvimento desse sistema, e que apresentaram um dos termos de busca no título, no resumo ou nas palavras-chave. Foram excluídos resultados que repetem um mesmo caso de aplicação, que apresentam altos níveis de semelhança, e/ou em que o objetivo não esteja diretamente relacionado com o tema de estruturas recíprocas na construção civil, deixando apenas uma amostra de cada um desses resultados.

Como estratégias de extração de dados foram estipuladas categorias para agrupamento das publicações, a partir do conteúdo presente nos resumos, introduções e conclusões dos trabalhos. A forma de classificação das publicações e a relevância dos dados coletados são apresentadas no item "Resultados".

\section{Condução}

Durante a condução, o termo "reciprocal frame" desvinculado de outros termos de busca foi satisfatório em quase todas as bases. Entretanto, quando aplicado no Periódicos CAPES, retornou um número muito elevado de resultados. Por isso, somente nessa base foi necessário utilizar os termos compostos para filtrar os trabalhos. O Quadro 1 sintetiza o processo de obtenção e de refinamento dos resultados obtidos pela pesquisa, com um total de 49 publicações para comunicação e análise.

\section{Resultados}

Após a definição dos trabalhos para análise e extração de dados, as publicações foram divididas em dois grupos, definidos pela presença ou não do desenvolvimento de produtos físicos nos trabalhos. Para cada um desses grupos foram definidas subcategorias que explicitam o assunto geral abordado.

Um grupo foi denominado como desenvolvimentos teóricos, constituído pelos subgrupos de:

(a) discussão, com artigos focados em aspectos históricos sobre o uso das estruturas recíprocas ou revisões sobre o tema, com as possibilidades futuras de aplicação;

(b) geometria, com estudos e pesquisas sobre as diversas possibilidades geométricas das ERs, podendo ou não apresentar a modelagem virtual de estruturas; e

(c) engenharia, com o foco voltado para a discussão de cálculo estrutural, detalhamento das conexões estruturais e modelos virtuais para simulações de desempenho.

Outro grupo foi chamado de aplicações práticas e é composto pelas subcategorias de:

(a) prototipagem, que contempla os trabalhos que apresentaram a produção de protótipos em escala 1:1 ou instalações físicas, fabricadas digitalmente; e 
(b) ensino, que se refere às experiências de ensino de alunos de graduação ou pós-graduação por meio de workshops, que também teve a produção de modelos físicos, principalmente em escala reduzida.

Após o agrupamento dos artigos por subcategorias, eles foram distribuídos conforme o ano de publicação. Dessa forma, é possível visualizar a evolução dos estudos e identificar as possíveis tendências e correlações entre as pesquisas e os autores (Figura 2).

A partir de 2007, é possível notar a publicação de, no mínimo, um artigo sobre o tema por ano (com exceção de 2012). Estudos que relatam experiências práticas aparecem apenas a partir de 2011. Existem alguns tópicos recorrentes em quase todos os artigos, independentemente da categoria. Um deles é a apresentação de uma definição mais ou menos detalhada do que são estruturas recíprocas, enquanto outro é a apresentação histórica sobre esse tipo de estrutura.

Dito isso, apresenta-se a seguir uma síntese dos assuntos abordados em cada uma das categorias e subcategorias, garantindo uma noção mais global dos estudos realizados.

Quadro 1 - Condução da RSL - Síntese dos procedimentos para definição da amostra

\begin{tabular}{|c|c|c|c|c|c|c|c|}
\hline $\begin{array}{c}\text { Parâmetro de } \\
\text { busca }\end{array}$ & \multicolumn{5}{|c|}{ Aplicações de busca } \\
\hline $\begin{array}{c}\text { Formas de } \\
\text { publicação }\end{array}$ & Periódicos & Congressos & \multicolumn{5}{|c|}{ Periódicos ou congressos } \\
\hline Bases de dados & $\begin{array}{c}\text { Periódicos } \\
\text { CAPES }\end{array}$ & CumInCAD & $\begin{array}{c}\text { Sage } \\
\text { Journals }\end{array}$ & $\begin{array}{c}\text { Ingenta } \\
\text { Connect }\end{array}$ & Compendex & SCOPUS & $\begin{array}{c}\text { Web of } \\
\text { Science }\end{array}$ \\
\hline Total por base & 30 & 8 & 25 & 12 & 29 & 53 & 22 \\
\hline $\begin{array}{c}\text { Total de } \\
\text { publicações }\end{array}$ & \multicolumn{7}{|c|}{180} \\
\hline $\begin{array}{c}\text { Eliminação por } \\
\text { repetição }\end{array}$ & \multicolumn{7}{|c|}{80} \\
\hline $\begin{array}{c}\text { Eliminação por } \\
\text { critérios de } \\
\text { exclusão }\end{array}$ & \multicolumn{7}{|c|}{40} \\
\hline $\begin{array}{c}\text { Publicações para } \\
\text { extração de dados }\end{array}$ & \multicolumn{7}{|c|}{49} \\
\hline
\end{tabular}

Figura 2 - Gráfico de artigos publicados por ano, separados por subcategorias e linha de evolução no tempo dos trabalhos por categoria

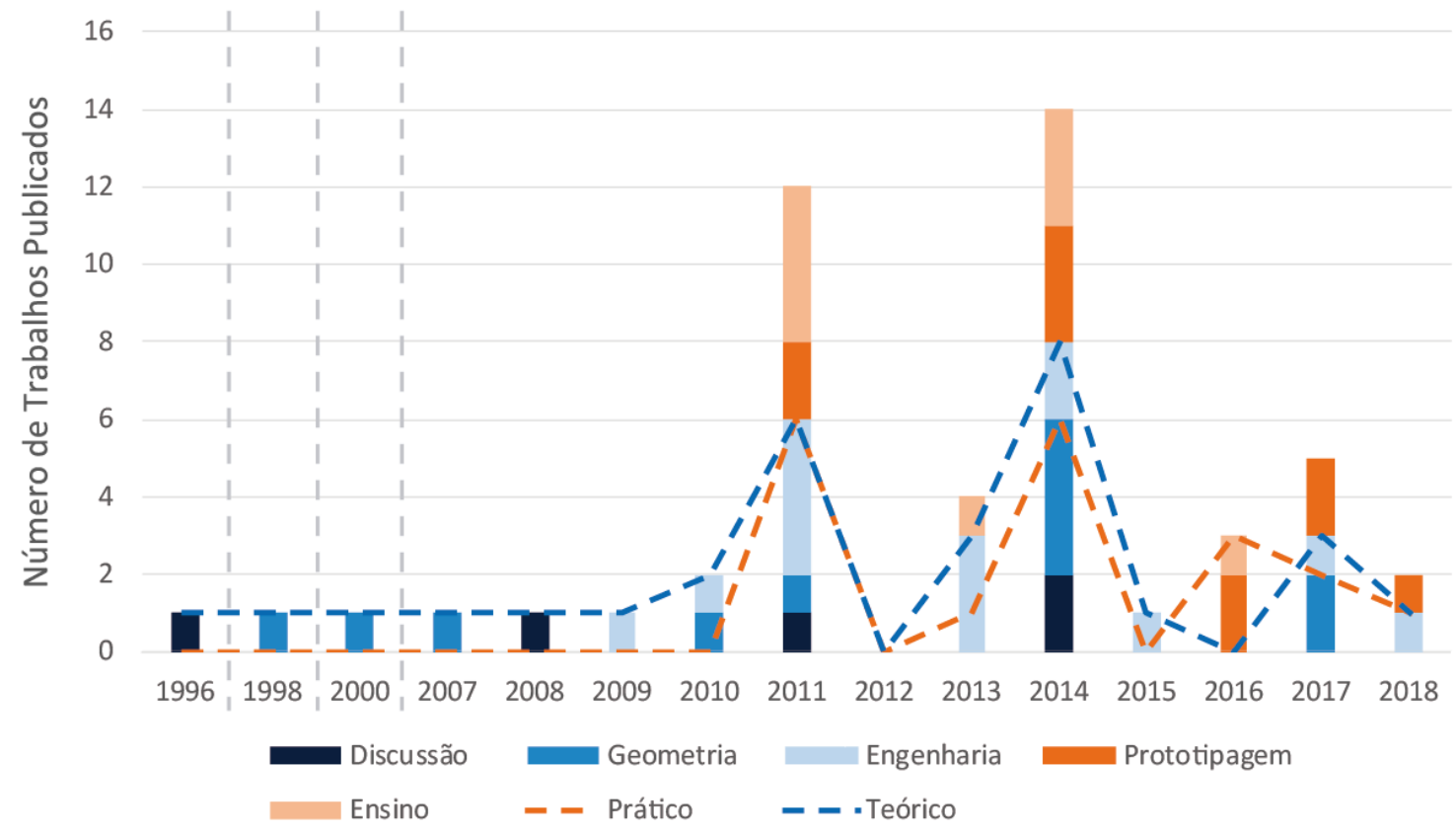




\section{Teoria}

\section{Discussão sobre ER}

Contempla um total de cinco artigos. Dois deles abordam os estudos feitos por Leonardo da Vinci sobre estruturas recíprocas e como podem contribuir para o desenvolvimento contemporâneo delas. Larsen, Chilton e Choo (1996) indicam algumas das possíveis vantagens e qualidades do uso dessas estruturas para a arquitetura contemporânea, com destaque para a possibilidade de se construir grandes vãos e configurações espaciais complexas, além da agilidade de montagem. Do ponto de vista conceitual, teórico e crítico, um dos trabalhos mais conceituados é o de Larsen (2014). Nele a autora apresenta algumas das experiências e inovações contemporâneas sobre o uso das estruturas recíprocas, ao mesmo tempo em que estabelece relações históricas, sendo as estruturas recíprocas uma forma de demonstrar avanços tecnológicos consideráveis. O último artigo nesta subcategoria é referente a uma revisão crítica sobre a os estudos e aplicações de estruturas recíprocas, elaborado por Pugnale e Sassone (2014).

\section{Geometria}

Foram encontrados 11 artigos, que ilustram os avanços da interpretação formal das estruturas recíprocas. Os artigos publicados no final da década de 1990 contemplam discussões sobre as possibilidades formais das estruturas recíprocas, sendo apresentados quase como um resgate histórico. Existem diversas tentativas de classificação dessas estruturas, inicialmente observadas no trabalho de Saidani, Baverel e Cross-Rudkin (1998), que apresentam o conceito de sistemas multirrecíprocos como alternativa aos sistemas em grelha convencionais. Baverel et al. (2000) definem o termo "Nexorade", com o intuito de compreender a composição geométrica e estrutural de todos os nós que conformam as estruturas recíprocas - um desdobramento da ideia dos sistemas multirrecíprocos. Baverel e Nooshi (2007) retomam o termo e sistematizam um método de produção de estruturas baseadas em poliedros regulares. Baverel e Larsen (2011) apresentam o termo woven structure (estrutura entrelaçada), como um tipo de estrutura que engloba as estruturas recíprocas (ou nexorades), em malha (ou tricô), em tecido (ou trançado) e em trabalho de cesta (ou canasta). Baverel e Pugnale (2014) procuram classificar essas estruturas de forma similar. Tamke, Riiber e Jungjohann (2010) combinam técnicas tradicionais de carpintaria com processos de fabricação digital. O estudo sobre a ampliação do Euskalduna Conference Centre and Concert Hall, feito por Bernabeu Larena e García Ménendez (2014), exemplifica e discute a produção contemporânea de estruturas recíprocas. Barrallo, González-Quintial e Sánchez-Parandiet (2017) indicam desdobramentos sobre os elementos que compõem as estruturas recíprocas. Gherardini e Leali (2017) sugerem o método de modelagem bottom-up, a partir dos elementos singulares até a composição da estrutura completa para alcançar soluções mais flexíveis, otimizadas (dado determinado objetivo), e geometrias ainda mais complexas e interessantes. Os estudos de Parigi et al. (2014) apresentam a teoria que sustenta a criação de uma ferramenta digital denominada "Reciprocalizer", apresentada na publicação de Parigi e Kirkegaard (2014a), que realiza processos iterativos para encontrar as soluções mais adequadas, sob o aspecto formal-estrutural. Resultados dessa pesquisa podem ser vistos nos trabalhos de Parigi e Pugnale (2014) e Parigi e Kirkegaard (2014b) na subcategoria Ensino.

\section{Engenharia}

Esta é a subcategoria com o maior número de trabalhos publicados, com um total de 14 artigos. Um dos assuntos recorrentes acerca das estruturas recíprocas é o desenvolvimento e aprimoramento de métodos de cálculo, com o intuito de solucionar a complexidade mecânica dessas estruturas. Douthe e Baverel (2009) exploram as vantagens sobre a aplicação do método de relaxamento dinâmico para o projeto de estruturas recíprocas por form-finding ${ }^{l}$. Já o artigo de Brocato (2011) apresenta as principais condições para que uma estrutura recíproca possua indeterminação estática. O estudo de Kohlhammer e Kotnik (2011) contém um método de análise que estabelece, matematicamente, a interação entre as forças atuantes no sistema global com todos os subsistemas presentes em uma estrutura recíproca. Para verificar o comportamento estrutural, Gelez, Aubry e Vaudeville (2011b) aplicam dois métodos analíticos das forças atuantes em uma grelha convencional e uma estrutura recíproca em composição similar, bidimensional. Garavaglia et al. (2013)

\footnotetext{
1De modo geral, os métodos de form-finding estão comumente associados ao uso de análises por elementos finitos e são utilizados com dois objetivos principais: (a) nos casos em que as ERs consideram a sobreposição de barras e uma geração iterativa, como a ferramenta reciprocalizer de Parigi e Kirkegaard (2014a) ou (b) para a obtenção de formas funiculares, que geram estruturas mais eficientes aos esforços gravitacionais, como pode ser visto nos trabalhos de Douthe e Baverel (2009) e Nabaei, Bavarel e Weinand (2013).
} 
utilizam métodos analíticos e numéricos que indicam que o comportamento de colapso das ERs é similar ao de vigas "tradicionais" conectadas entre si, porém com menor robustez. Nabaei, Baverel e Weinand (2013) aplicam o método de relaxamento dinâmico para obter a disposição dos elementos estruturais na geometria global de uma ER composta por Timber Fabric. Por sua vez, Mesnil et al. (2018) adotaram o translation method (método de translação) para o form-finding de estruturas recíprocas. Rizzuto e Larsen (2010) apresentam uma síntese dos métodos tradicionais e contemporâneos de conexões utilizadas nessas estruturas. Nabaei e Weinand (2011) exploram o conceito de placas dobradas, substituindo os elementos lineares convencionais, e analisam as deformações na estrutura. Kohlhammer et al. (2017) relacionam a geometria e o comportamento estrutural de estruturas recíprocas, explorando o uso de conexões em T coladas, com a montagem auxiliada por robôs. Song et al. (2013) desenvolveram uma ferramenta utilizando C++ e OpenGL, capaz de alocar diversos padrões geométricos em diferentes superfícies, com auxílio de novos métodos de form-finding desenvolvidos pelos pesquisadores. Algumas das limitações encontradas foram resolvidas por Song et al. (2014), como o aprimoramento da interface do usuário, resultados mais precisos e incorporação do processo de fabricação de estruturas recíprocas. A ferramenta digital de form-finding desenvolvida por Thonnissen (2014) tem o objetivo de fazer com que as estruturas recíprocas sejam mais acessíveis e fáceis de projetar. Garcia Puyol (2015) sugere um fluxo de projeto que se beneficia da customização em massa, com o uso dos softwares Rhinoceros e Grasshopper, aprimorados pelos plug-ins Kangaroo, Millipede, HAL e componentes customizados em C\#.

\section{Prática}

\section{Ensino}

Foram alocados nesta subcategoria nove artigos. Dentre eles, quatro são respectivos a relatos de workshops publicados no quarto número do International Journal of Space Structures em 2011. Todos indicam que a experimentação prática contribui para a compreensão do comportamento e da lógica das estruturas recíprocas (GELEZ; SABY, 2011; BAVEREL et al., 2011; LARSEN, 2011; THONNISSEN; WERENFELS, 2011; LARSEN; LEE, 2013; AVELLANEDA et al., 2014; PARIGI; PUGNALE, 2014; PARIGI; KIRKEGAARD, 2014a, 2014b; GUTIERREZ; FLORES; PRECIADO, 2016). No entanto, é importante lembrar que seria impossível simplesmente extrapolar o comportamento estrutural de um modelo em escala para uma obra no âmbito da construção civil. É necessário readequar o dimensionamento para a nova escala, incorporar as características dos materiais e os processos de fabricação e montagem.

\section{Prototipagem}

Fazem parte desta subcategoria 10 artigos que apresentam a construção de estruturas recíprocas, como provas de conceito, resultados de pesquisa ou obras reais. O dodeca-icosahedron de Sénéchal, Douthe e Baverel (2011) foi a aplicação prática de métodos analíticos de form-finding e de transformações geométricas pesquisados pelos autores, montado por duas pessoas em apenas quatro horas. Gelez, Aubry e Vaudeville (2011a) descrevem a obra vencedora do concurso para o abrigo arqueológico de Bibracte, na França. O artigo de Maxwell et al. (2014) descreve o projeto e a construção da estrutura recíproca do pavilhão Utzon/40, que combina dois tipos de materiais, processos de form-finding e o fluxo de produção file-to-factory. O protótipo feito por Pantazis e Gerber (2014) foi composto por chapas curvas de madeira compensada, concebido pela combinação de técnicas de form-finding e multi agent systems como estratégias generativas bottom-up. Sánchez-Sánchez, Escrig Pallarés e Rodríguez-León (2014) se basearam no conceito de reciprocidade para desenvolver uma estrutura recíproca fractal semelhante à de uma árvore para o Natural Interpretation Centre de Melilla, no norte da África. A pesquisa promovida por Tong e Zhou (2016) aponta que, apesar da maior complexidade presente nas estruturas recíprocas tridimensionais, sua criação está intrinsecamente relacionada com os dados de entrada que definem as regras de crescimento, as dimensões dos elementos lineares e a geometria inicial. Já a proposta de Anastas, Rhode-Barbarigos e Adriaenssens (2016) é a de solucionar a geração de estruturas recíprocas adaptáveis a diversas superfícies a partir da subdivisão de meshes, com o auxílio de um algoritmo baseado em padrões celulares. O projeto experimental do pavilhão DigDesFab15 de Gheorghe e Vierlinger (2017) resultou na criação de uma estrutura recíproca que utiliza um polímero de concreto para conectar os elementos de madeira. Com o objetivo de investigar a aplicação das estruturas recíprocas como uma tipologia arquitetônica contemporânea, Araullo e Haeusler (2017) desenvolveram um sistema de encaixes duplos assimétricos para efetivar as conexões entre peças planas. Araullo (2018) tenta estabelecer os critérios que devem ser considerados para compor a morfologia tridimensional (uvw) das estruturas recíprocas por meio da construção do protótipo Mobius, baseado em uma parte da fita de Mobius. 


\section{Análise do conteúdo encontrado}

A partir dos dados extraídos, foi possível identificar, em linhas gerais, as principais convergências e divergências entre os autores. $\mathrm{O}$ material analisado permitiu, em primeiro lugar, sistematizar as diferentes definições e nomenclaturas dadas às estruturas recíprocas. Também foi possível identificar diferentes tipos de classificação dessas estruturas, segundo critérios variados. A grande maioria dos artigos também forneceu informações históricas. Embora não seja possível, a partir delas, traçar uma trajetória contínua sobre o emprego dessas estruturas, foi possível inferir a relação entre o uso das estruturas recíprocas e os momentos de grande desenvolvimento tecnológico. Finalmente, essa análise permitiu identificar lacunas do conhecimento a serem preenchidas. Dentre os 49 artigos, 27 afirmam existir espaço para pesquisas e inovações, sugerindo desdobramentos ou estudos futuros.

\section{Definições}

O primeiro tópico que deve ser esclarecido para compreender as estruturas recíprocas está na nomenclatura utilizada para se referir a esse tipo de estrutura. O trabalho de Pugnale e Sassone (2014) apresenta um compilado dos termos utilizados de forma científica para se referir a esse tipo de estrutura, sendo eles: "Mandala Roof", "Reciprocal Frame", "Serlio Floor", "Multi-Reciprocal Grid", "Nexorade", "Leverworks", "Multi-Reciprocal Element", "Mutually Supported Element", "Leonardo's Lattice", "Selfsupporting Framework", "Lamella Flock" e "Interlocking Frame". Outros termos como "Reciprocal System" ou "Zollinger System" aparecem em alguns artigos, que não foram analisados pelos autores. Há também algumas pequenas variações, como o uso de "Reciprocal Structure" no lugar de "Reciprocal Frame". Todavia, os principais autores (com maior número de publicação e/ou publicações mais citadas) e os artigos mais recentes têm utilizado "Reciprocal Frame" ou "Nexorade", o que pode indicar uma concordância sobre quais os termos considerados mais adequados ou corretos.

O conceito fundamental dessas estruturas encontra-se na relação entre "suportar e ser suportado". Conforme Pugnale e Sassone (2014), elas possuem duas características inerentes:

(a) todos os elementos atuam como suporte e como suportados simultaneamente e simetricamente; e

(b) essas duas funções não são sobrepostas no mesmo ponto do elemento estrutural, e não configuram uma hierarquia estrutural.

Outra propriedade mencionada por Larsen (2014) é o fato dos componentes serem curtos e lineares ou bidimensionais, capazes de configurar sistemas complexos e fechados. Por sua vez, Baverel et al. (2000) afirmam que os elementos individuais, ou "nexors", sempre possuem quatro regiões de conexão, estabelecidas entre dois nexors diferentes. Baverel e Pugnale (2014) acrescentam que essas regiões sempre se dão ao longo do elemento e nunca nas extremidades laterais (Figura 3).

A região de encontro estabelecida por no mínimo três componentes é considerada como o padrão geométrico elementar, também chamado de leque pela maioria dos autores. Mais recentemente, Araullo (2018) sugere o termo "DNA" como uma analogia biológica, uma vez que as ferramentas digitais permitem a personalização em massa das geometrias que configuram determinada estrutura. Cada nexor apresenta determinada angulação e uma dimensão de trabalho. O perímetro gerado entre essas dimensões forma a área chamada de janela de trabalho. Além disso, a configuração espacial gerada pelos leques implica a sobreposição da excentricidade dos nexors, dada como a menor distância entre o eixo de dois elementos conectados (BAVEREL et al., 2000; DOUTHE; BAVEREL, 2009; SÉNÉCHAL; DOUTHE; BAVEREL, 2011; THONNISSEN, 2014; MESNIL et al., 2018) (Figura 4).

Quase todos os autores levantados concordam que um mecanismo-chave para viabilizar a produção e a montagem de qualquer tipo de estrutura recíproca é a forma de conexão entre os elementos. De modo geral, Rizzuto e Larsen (2010) descrevem que elas podem ser feitas por atrito e pressão, por encaixes ou auxiliadas por fixadores mecânicos (parafusos) ou químicos (colas). No estudo sobre o uso de conexões em T, Kohlhammer et al. (2017) indicam que a região e a geometria de contato entre os nexors são dois critérios que têm uma grande influência sobre a eficiência da conexão adotada, além de sugerirem que a redução da excentricidade entre os elementos pode melhorar o desempenho estrutural. Araullo e Haeusler (2017) indicam que, nas estruturas compostas tanto por elementos lineares como por bidimensionais, as especificidades encontradas nas áreas individuais de conexão demandam um projeto completamente novo e que, em geral, o uso de produtos disponibilizados pelo mercado, de forma padronizada, é insuficiente para suprir as necessidades de todas as conexões. 
Quanto aos aspectos estruturais, é possível afirmar que os principais esforços atuantes sobre as estruturas recíprocas são esforços axiais e esforços de flexão e as principais estratégias adotadas para verificar o comportamento estrutural utilizam análise por elementos finitos. De acordo com Mesnil et al. (2018), esse tipo de estrutura apresenta comportamentos muito peculiares, que devem ser tratados de forma não convencional. O estudo de Brocato (2011) sugere que a otimização ${ }^{2}$ estrutural em nexorades pode ser obtida pela redução das forças axiais e aumento das de flexão, o que é oposto ao que usualmente é feito em estruturas em cascas ou gridshell. Por outro lado, Kohlhammer e Kotnik (2011) sugerem que, ao decompor a estrutura em seus elementos individuais, é possível analisar cada um como vigas tradicionais. O comportamento de colapso desse tipo de estrutura também se aproxima de qualquer estrutura "tradicional" formada por vigas conectadas, submetida aos mesmos tipos de esforços, como mencionado por Garavaglia et al. (2013).

Figura 3 - Representação em perspectiva das áreas de conexão de um nexor EIXO DE SIMETRIA

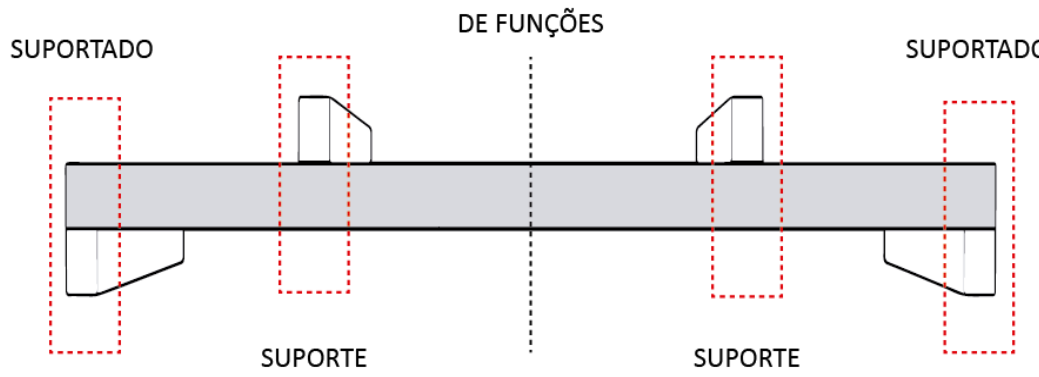

REGIÕES DE CONEXÃO

NEXOR ANALISADO

NEXORS CONECTADOS

Figura 4 - Propriedades existentes em um leque de uma estrutura recíproca, exemplificado por um leque triangular

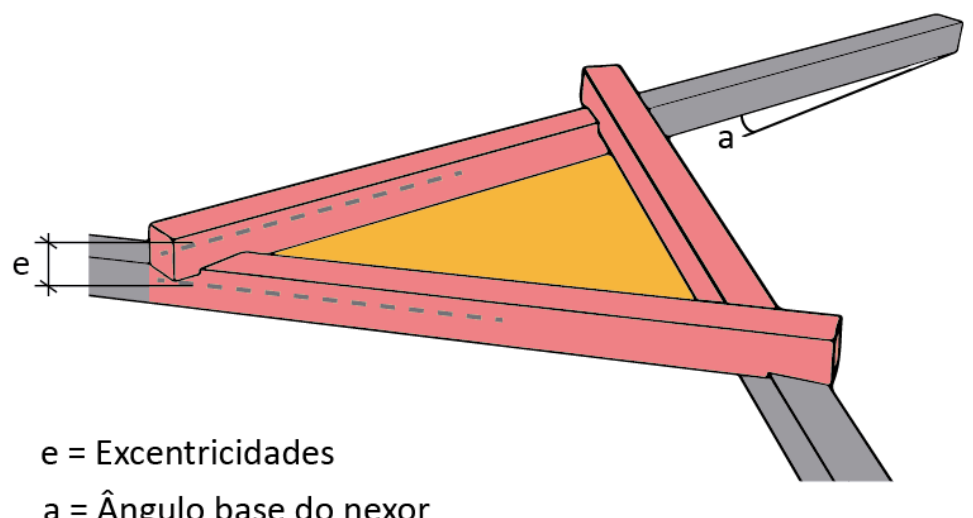

$a=$ Ângulo base do nexor

- - - Eixo central do nexor

Dimensão de trabalho

Janela de trabalho

\begin{abstract}
${ }^{2} \mathrm{~A}$ otimização estrutural de ERs pode ser realizada de diversas formas. Por exemplo, ela estar relacionada com a geometria e o tipo de seção transversal das barras que compõem as ERs ou com a forma global da estrutura. No caso de Brocato (2011), o processo de otimização foi feito pela relação entre a geometria da estrutura e a disposição de nexors apoiados sem o uso de fixadores mecânicos ou químicos.
\end{abstract}




\section{Classificação}

Classificar os tipos de estruturas recíprocas pode ser considerada como uma ação desafiadora, sobretudo por causa das suas peculiaridades inerentes. De modo geral, diversos autores mencionam que seis itens são analisados para categorizar essas estruturas (BAVEREL; LARSEN, 2011; BAVEREL; PUGNALE, 2014; THONNISSEN, 2014):

(a) a geometria dos leques;

(b) o número de elementos que se conectam;

(c) a dimensão de trabalho;

(d) a excentricidade entre as peças;

(e) o comprimento total de uma peça; e

(f) a configuração topológica da estrutura.

Baverel e Larsen (2011) consideram a existência de um grupo denominado como estruturas entrelaçadas, que engloba quatro subcategorias. Dentre elas, as estruturas recíprocas são aquelas compostas por elementos descontínuos que apresentam resistência elevada ou mediana à flexão. Elas ainda são divididas em quatro subgrupos, conforme o tipo de elemento utilizado, sendo eles:

(a) alongadas ou lineares: podem ser caracterizadas de acordo com a geometria dos leques, a disposição final, a dimensão de comprometimento e a excentricidade;

(b) anéis: exemplificadas pelos anéis de Borromé e Hopf;

(c) planos (placas ou lâminas): diferenciados pela geometria e pela disposição dos elementos utilizados, principalmente se houver algum tipo de dobra ou curvatura; e

(d) blocos - caracterizadas por apresentar dimensões semelhantes em três dimensões e é por ser a única situação em que os leques são compostos por apenas dois elementos, sendo reconhecidos como "elementos tridimensionais".

Entretanto, autores como Pugnale e Sassone (2014) não concordam com a possibilidade de se utilizar esse quarto subgrupo para a conformação de estruturas recíprocas, uma vez que o comportamento estrutural se aproxima mais de um sistema em casca; para existir reciprocidade, é necessário que a relação entre ação e reação de dois elementos seja perfeitamente simétrica. Caso contrário, trata-se apenas de um benefício mútuo entre elementos.

Figura 5 - Estrutura do pavilhão Serpentine Gallery de 2005

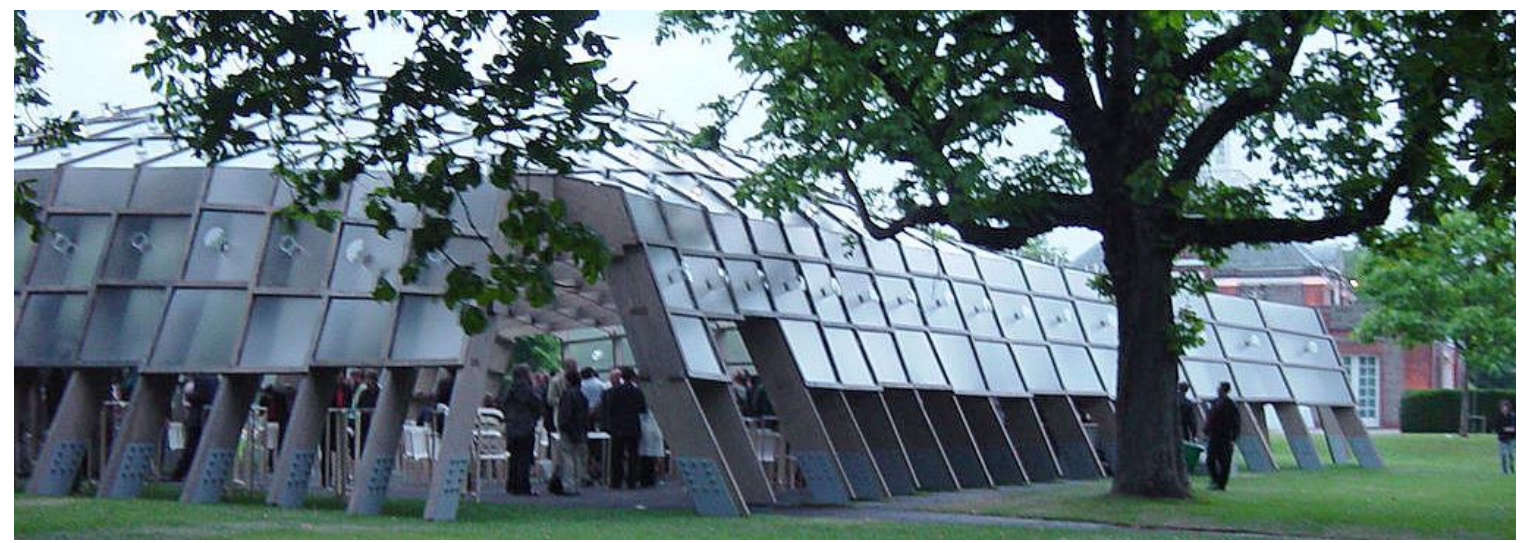

Fonte: INCITE Researchers (2005). 
Baverel e Pugnale (2014) apresentam uma tentativa de classificação das estruturas recíprocas compostas por elementos planos. São definidos cinco grupos distintos, sendo eles:

(a) elementos planos usados como elementos lineares espessos: estruturas compostas por elementos planos que substituem os elementos lineares, exemplificados pela Serpentine Gallery (Figura 5), de Álvaro Siza, Eduardo Souto de Moura e Cecil Balmond;

(b) elementos planos usados como agrupamentos de elementos lineares: estruturas em que os elementos planos substituem os leques formados por elementos lineares;

(c) elementos planos que transmitem momento pelas conexões: estruturas que não são baseadas na formação de leques e são estabilizadas pela maximização na transmissão do momento gerado pelos esforços mecânicos;

(d) elementos planos que transmitem tração e compressão pelas conexões: estruturas que permitem a transmissão das forças de tração e compressão pelas conexões com características estruturais similares as de uma treliça convencional, e também não se baseiam na formação de leques; e

(e) combinação de características apresentadas nas quatro categorias anteriores.

Outra forma de diferenciar as estruturas recíprocas é pelo tipo de método utilizado para a obtenção da forma. As pesquisas apresentadas demonstram a aplicação de diferentes técnicas de análise por elementos finitos combinadas com form-finding, com destaque para o método do relaxamento dinâmico (DOUTHE; BAVEREL, 2009), e o método de translação (MESNIL et al., 2018).

\section{Lacunas identificadas}

Com base nos 27 artigos que identificaram explicitamente algum tipo de lacuna do conhecimento sobre as estruturas recíprocas, foi possível notar cinco tópicos recorrentes que demandam aprofundamento em pesquisas e estudos:

(a) dificuldades na concepção geométrica/estrutural;

(b) necessidade de aprimoramento de ferramentas digitais;

(c) dificuldade no projeto, análise, fabricação e montagem de conexões;

(d) dificuldade de compreensão da real contribuição dos protótipos para aplicações em grande escala; e

(e) necessidade de incorporar o processo de montagem no projeto de ERs.

As lacunas estão relacionadas com os artigos identificados e seus respectivos autores no Quadro 2. Em seguida, com base nas informações obtidas, é apresentado um encadeamento lógico que relaciona declarações feitas pelos diferentes autores. É importante mencionar que alguns artigos indicam mais de um tipo de lacuna; entretanto, optou-se por focar nos aspectos mais destacados em cada artigo.

\section{Quadro 2 - Principais lacunas de conhecimento sobre estruturas recíprocas e os artigos em que foram} encontradas

\begin{tabular}{|c|c|}
\hline Lacunas identificadas & Artigos \\
\hline $\begin{array}{c}\text { Dificuldades na concepção } \\
\text { geométrica/estrutural }\end{array}$ & $\begin{array}{c}\text { Baverel e Pugnale (2014); Baverel e Larsen (2011); Tong e Zhou (2016); } \\
\text { Kohlhammer } \text { et al. (2017); Gelez, Aubry e Vaudeville (2011b); e } \\
\text { Barrallo, González-Quintial e Sánchez-Parandiet (2017). }\end{array}$ \\
\hline $\begin{array}{c}\text { Necessidade de } \\
\text { aprimoramento de } \\
\text { ferramentas digitais }\end{array}$ & $\begin{array}{c}\text { Garcia Puyol (2015); Nabaei, Baverel e Weinand (2013); Mesnil et al. } \\
(2018) ; \text { Garavaglia } \text { et al. (2013); Kohlhammer e Kotnik (2011); } \\
\text { Gherardini e Leali (2017); e Thonnissen (2014). }\end{array}$ \\
\hline $\begin{array}{c}\text { Dificuldade no projeto, } \\
\text { análise, fabricação e } \\
\text { montagem de conexões }\end{array}$ & $\begin{array}{c}\text { Maxwell et al. (2014); Nabaei e Weinand (2011); Parigi et al. (2014); } \\
\text { Rizzuto e Larsen (2010); e Saidani, Baverel e Cross-Rudkin (1998). }\end{array}$ \\
\hline $\begin{array}{c}\text { Dificuldade de extrapolação } \\
\text { de protótipos para aplicações } \\
\text { em grande escala }\end{array}$ & $\begin{array}{c}\text { Gutierrez, Flores e Preciado (2016); Parigi e Pugnale (2014); Gheorghe } \\
\text { e Vierlinger (2017); e Araullo e Haeusler (2017). }\end{array}$ \\
\hline $\begin{array}{c}\text { Necessidade de incorporar o } \\
\text { processo de montagem no } \\
\text { projeto de ERs }\end{array}$ & $\begin{array}{c}\text { Larsen, Chilton e Choo (1996); Larsen e Lee (2013); Araullo (2018); } \\
\text { Bernabeu Larena e García Ménendez (2014); e Song et al. (2013). }\end{array}$ \\
\hline
\end{tabular}


Dificuldades na concepção geométrica/estrutural (6 artigos): a investigação e sistematização das abordagens feitas sobre as ERs é importante para a compreensão das diversas variáveis que influenciam sua construção, desde morfológicas até o detalhamento e processo de montagem. Esse tipo de trabalho investigativo também pode demonstrar "[...] a necessidade de aprofundar explorações geométricas, estruturais e construtivas [...]" (BAVEREL; PUGNALE, 2014, p. 188, tradução nossa). Além disso, a geometria e o tipo dos nexors e dos leques implicam configurações complexas que demandam pesquisas adicionais, com a proposta de encontrar "[...] uma abordagem unificada para descrever claramente os sistemas recíprocos [...]” (BAVEREL; LARSEN, 2011, p. 288, tradução nossa).

Do ponto de vista da composição e modelagem geométrica, mesmo os casos mais complexos, como os de ERs tridimensionais (com crescimento em uvw), podem seguir lógicas de modelagem similares às de uma estrutura paramétrica típica. Entretanto, é necessário que o conjunto de regras geradoras das ERs seja explícito e bem definido. Para ilustrar, “[...] o experimento ainda utiliza regras simples de desenvolvimento. As regras complicadas não foram implementadas. Se for possível utilizar diferentes regras no processo de geração, o resultado pode se tornar mais complexo e interessante [...]" (TONG; ZHOU, 2016, p. 734, tradução nossa). Pode-se entender que essas regras mais complicadas estão, muitas vezes, associadas a uma relação complexa e singular estabelecida entre a composição geométrica e o desempenho estrutural. Pequenas variações em ângulos ou dimensões nos nexors podem exercer grande influência na estabilidade global dessas estruturas. Nesse sentido, “[...] as configurações ideais dos parâmetros geométricos em relação à capacidade estrutural não são evidentes nem fáceis de derivar. Contudo, esses parâmetros podem ser otimizados com o auxílio de ferramentas computacionais [...]" (KOHLHAMMER et al., 2017, p. 214, tradução nossa). O artigo de Gelez, Aubry e Vaudeville (2011b) não só ilustra como essas relações podem ocorrer em uma aplicação específica de ER como reforça as lacunas existentes ao afirmar que "[...] é necessário o desenvolvimento trabalhos adicionais para entender o efeito de cargas e geometrias mais complexas no comportamento estrutural apresentado [...]" (GELEZ; AUBRY; VAUDEVILLE, 2011b, p. 342, tradução nossa).

Uma alternativa para solucionar problemas recorrentes das ERs, como a forma de vedação, pode ser feita pela exploração de nexors dobráveis, curvos ou maleáveis. Mesmo sendo um caminho interessante, como é demonstrado em estudos de chapas curvas maleáveis, ele implica a necessidade de "[...] analisar profundamente o comportamento estrutural das chapas curvas não-rígidas nas construções [...]" (BARRALLO; GONZÁLEZ-QUINTIAL; SÁNCHEZ-PARANDIET, 2017, p. 738, tradução nossa).

Os principais tópicos que sintetizam esta lacuna são:

(a) as regras que geram ERs de forma paramétrica são complexas e não bem definidas;

(b) no é claro como o desempenho estrutural é influenciado por cada tipo de variação geométrica, seja nos nexors, nos leques ou na forma global; e

(c) considerar sistemas acoplados ao sistema estrutural implica problemas não bem definidos, como é o caso da vedação.

Necessidade de aprimoramento de ferramentas digitais (7 artigos): o uso de ferramentas digitais no processo de projeto de ERs é utilizado por quase todos os autores, seja para a modelagem da estrutura, para análises estruturais e/ou para fabricação de componentes personalizados e complexos. Para Garcia Puyol (2015) a principal limitação de seu trabalho é a exigência de muito poder de processamento computacional, o que acarreta um longo tempo gasto para o funcionamento do algoritmo de modelagem geométrica paramétrica. Isso ocorre sem considerar as características específicas da madeira (como a variação dimensional heterotrópica e a direção das fibras) ou uma análise detalhada individual das peças para montagem; a implementação dessas variáveis implicaria um processo ainda mais lento. Paralelamente, Nabaei, Baverel e Weinand (2013) afirmam que as simulações por métodos de elementos finitos também são muito dispendiosas, apesar de terem uma elevada precisão, quando aplicadas a geometrias não lineares. $\mathrm{O}$ desenvolvimento de novos métodos de análise por elementos finitos para a geração e otimização da estrutura, aliadas aos princípios de form-finding, encontram limitações em atender todas as demandas das ERs. Um exemplo é o desenvolvido por Mesnil et al. (2018), que apesar de conseguir solucionar uma categoria específica de problemas, sugere que "[...] o método poderia ser acoplado aos métodos existentes de form-finding, como o relaxamento dinâmico, a fim de satisfazer todas as restrições de excentricidades [...]" (MESNIL et al., 2018, p. 152, tradução nossa).

Para Garavaglia et al. (2013, p. 545) "[...] o desenvolvimento de pesquisas futuras e adicionais devem se preocupar com a otimização da forma e dos materiais, para uma aplicação confiável das ERs [...]” (tradução 
nossa). Para que isso ocorra, será necessário incluir variáveis dos materiais para a realização de modelagens e análises estruturais digitais mais precisas. Métodos desenvolvidos para análises estruturais de sistemas complexos também não utilizam, de forma efetiva, as variáveis do material. Isso pode ser observado na afirmação: “O desafio aqui é a indeterminação estática dos subsistemas. As forças de suporte [...] não podem mais ser determinadas apenas com geometria, [...] são necessárias premissas adicionais, como as de materiais ou de conexões [...]" (KOHLHAMMER; KOTNIK, 2011, p. 86, tradução nossa).

Outra limitação do uso de sistemas computacionais é encontrada nos níveis de automação, que dificilmente conseguem contemplar todo o processo de geração das ERs. O trabalho feito por Gherardini e Leali (2017) consegue gerar estruturas em inúmeras geometrias, mas exige a intervenção manual do arquiteto para a definição do primeiro leque, além de não alcançar um nível de otimização ideal para os elementos tridimensionais. Paralelamente, alguns métodos são desenvolvidos com o objetivo de otimizar a forma da estrutura pelo seu processo de geração. A ferramenta desenvolvida por Thonnissen (2014) tem o objetivo de facilitar a aplicação de ERs por form-finding e sua produção por equipamentos de controle numérico, mas não automatiza todo o processo. Na realidade, ela auxilia o controle do usuário sobre algumas das complexas relações geométricas e estruturais, mas a alteração dos parâmetros não gera um produto acabado, pronto para a fabricação e montagem. Isso porque a “[...] integração de análises e otimizações estruturais (na ferramenta) ainda é um desafio a ser superado [...]” (THONNISSEN, 2014, p. 106, tradução nossa).

Os principais tópicos que sintetizam esta lacuna são:

(a) as estratégias de modelagem e análise demandam um tempo excessivo ou muito poder computacional;

(b) há uma dificuldade em implementar variáveis dos materiais como parâmetros para as análises estruturais;

(c) ainda não foram encontradas formas efetivas de se integrar processos generativos de projeto e de análise estrutural das ERs; e

(d) a automação dos processos digitais ocorre de forma parcial, sem contemplar todo o processo, desde a concepção até a fabricação e montagem.

Dificuldade no projeto, análise, fabricação e montagem de conexões (5 artigos): "O projeto de conexão entre vários elementos pode ser desafiador [...], mas não deveria ser um grande problema [...]" (SAIDANI; BAVEREL; CROSS-RUDKIN, 1998, p. 217, tradução nossa). Todavia, como já foi mencionado, o sistema de conexões das ERs é uma das partes mais importantes para a viabilização dessas estruturas. A forma como os nexors estão relacionados e fixados implica o desempenho estrutural, no processo de fabricação e no de montagem. Alguns edifícios contemporâneos feitos com ERs, principalmente no Japão, servem de exemplo para mostrar a diversidade de tipos de conexão possíveis entre os nexors. Todavia, "[...] investigações experimentais e numéricas devem ser realizadas a fim de fornecer informações complementares sobre o comportamento estrutural das conexões feitas com parafusos ou encaixes em ERs submetidas a uma maior quantidade de esforços [...]" (RIZZUTO; LARSEN, 2010, p. 255, tradução nossa). Sobre o impacto das conexões das ERs nas análises estruturais, o trabalho de Maxwell et al. (2014) descreve um sistema de conexão complexo que é fabricado digitalmente. Porém, ele ainda demanda a integração de análises estruturais por elementos finitos aplicadas a objetos tridimensionais (a geometria e o tipo de conexão) em tempo real (em vez dos modelos simplificados de linhas) com as ferramentas generativas. Para os autores, somente dessa forma seria possível aprimorar e otimizar as soluções de conexão encontradas.

Outra perspectiva pouco explorada para a geração de ERs é a utilização das conexões como um dos parâmetros iniciais do processo de projeto. Nesse sentido, o trabalho apresentado por Nabaei e Weinand (2011) pode ser mencionado como um exemplo. Trata-se de uma ER derivada da tecnologia de conexões desenvolvida pelos autores, e que pode inspirar uma nova família de estruturas. Porém, ainda é necessário aplicar o conceito desenvolvido em outras geometrias, com nexors curvos, uma vez que a geometria da conexão deve ser determinada com base na geração da estrutura com auxílio de métodos de form-finding. $\mathrm{O}$ artigo apresentado por Parigi et al. (2014) apresenta uma proposta de conexão, sendo uma extensão dos estudos de Pellegrino e Calladine sobre um tipo específico de articulação para a montagem de ERs. Apesar dos resultados iniciais positivos sobre a avaliação do número de articulações internas e externas, ainda é “[...] necessário trabalhos adicionais para completar as especificações de todas as possíveis condições de restrições, como a inclusão de articulações deslizantes [...]” (PARIGI et al., 2014, p. 58, tradução nossa).

Os principais tópicos que sintetizam esta lacuna são:

(a) dificuldade em prever o comportamento estrutural das conexões e de realizar análises precisas em cada uma; e 
(b) em geral, as conexões são resolvidas depois do processo de geração das ERs, sem aproveitar a oportunidade de utilizá-las como um dos parâmetros iniciais do projeto.

Dificuldade de compreensão da real contribuição dos protótipos para aplicações em grande escala (4 artigos): apesar da existência de ERs em grandes escalas, como no abrigo arqueológico de Bibracte, descrito por Gelez, Aubry e Vaudeville (2011a), ou na parte externa do observatório de Mount Rokko-Shidare (Figura 6), muitas das inovações ficam restritas a escalas reduzidas, em consequência de restrições (orçamentárias e de tempo) do ambiente acadêmico. Gutierrez, Flores e Preciado (2016) sugerem que a pesquisa feita por eles poderia ter uma continuação com a incorporação de princípios de biomimicry e biomimetics para a geração de ERs, e que essas estruturas sejam uma forma de "[...] ensinar estudantes sobre sistemas estruturais recíprocos e alterar paradigmas construtivos nas comunidades estudantis [...]" (GUTIERREZ; FLORES; PRECIADO, 2016, p. 9, tradução nossa). De fato, a construção de pequenos pavilhões utilizando ERs é uma prática relativamente comum e pode ser observada em diversos artigos analisados, mas que ainda são distantes da realidade arquitetônica. Como exemplo, pode-se mencionar a seguinte consideração: "Os sete protótipos [...] foram elaborados em um ambiente baseado em pesquisa, e o escopo pretendido foi de utilizálos como o ponto de partida para o desenvolvimento de variações do mesmo conceito e de alguma aplicação real no contexto arquitetônico [...]" (PARIGI; PUGNALE, 2014, p. 176, tradução nossa).

Figura 6 - Elemento externo do observatório no monte Rokko-Shidare

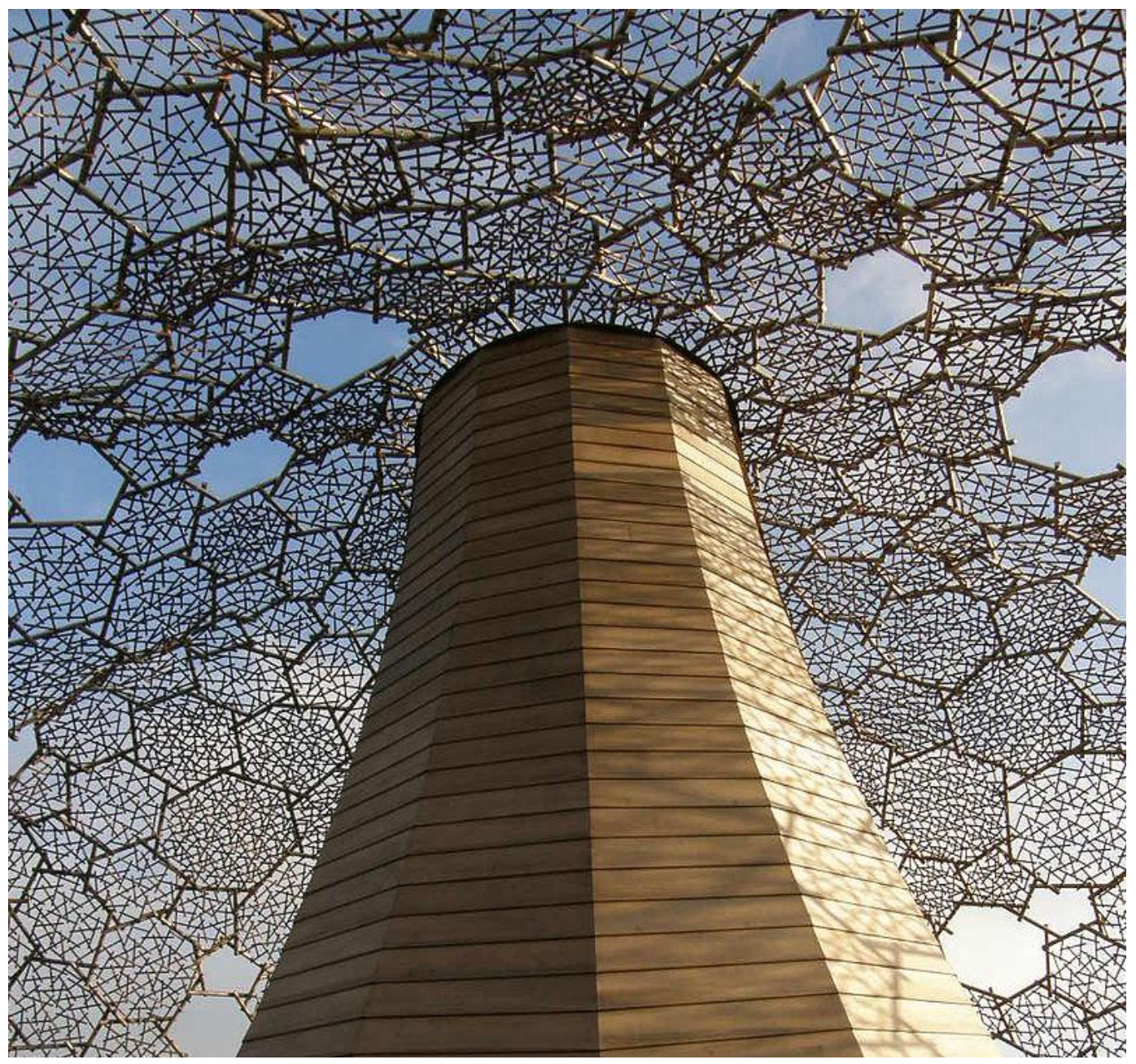

Fonte: Rokko-shidare (2020). 
Em outra experiência, o material composto desenvolvido por Gheroghe e Vierlinger (2017) foi também utilizado em uma ER em escala reduzida. Sua aplicação em grande escala demandaria “[...] um aprofundamento sobre avaliações estruturais do material composto desenvolvido e verificar a durabilidade a longo prazo, com exposição a intempéries (ultravioleta e umidade) [...]" (GHEORGHE; VIERLINGER, 2017, p. 7, art. 18, tradução nossa). Uma alternativa proposta por alguns autores para a aplicação das estruturas em maiores escalas seria de abordagens bottom-up, mas para isso é necessário "[...] incentivar pesquisas adicionais que levem as ERs (planares) dos laboratórios de pesquisa para o uso final como uma tipologia arquitetônica contemporânea de 'bricolagem' [...]” (ARAULLO; HAEUSLER, 2017, p. 548, tradução nossa).

Os principais tópicos que sintetizam esta lacuna são:

(a) há uma dificuldade em aproveitar efetivamente o conhecimento acadêmico desenvolvido em projetos na escala da construção civil; e

(b) as inovações desenvolvidas pelo conhecimento científico ficam, muitas vezes, restritas ao ambiente acadêmico, sem implementação efetiva na indústria.

Necessidade de incorporar o processo de montagem no projeto de ERs (5 artigos): de modo geral, é reconhecido que uma das principais vantagens das ERs é sua facilidade e agilidade de montagem. "A velocidade de construção de ERs em edifícios modulares permanentes é comparável ao de outros sistemas leves industrializados. Por outro lado, construções temporárias de ERs podem ser erguidas em um período relativamente curto [...]" (LARSEN; CHILTON; CHOO, 1996, p. 81, tradução nossa). Essa frase sugere a aplicação dessas estruturas para a produção de estruturas desmontáveis. Ainda mais interessante é a possibilidade de que essas estruturas podem ser aprimoradas de temporárias para permanentes, conforme é sugerido por Larsen e Lee (2013).

Entretanto, quando a complexidade geométrica aumenta e/ou é almejada para uma estrutura permanente, o processo de montagem se torna mais complexo e pode demandar profissionais mais especializados para sua execução. Isso pode ser exemplificado pelo sistema de montagem apresentado por Araullo (2018), que "[...] exige o uso da fabricação digital e pode ser beneficiado por processos CNC e de robótica avançada [...]" (ARAULLO, 2018, p. 316, tradução nossa). Mas é necessário considerar que alguns estudos detalhados sobre o processo de montagem apontam a necessidade de maiores "[...] definições e análises sobre os estágios intermediários da construção de estruturas recíprocas [...]” (BERNABEU LARENA; GARCÍA MÉNENDEZ, 2014, p. 67, tradução nossa). Esse também é um dos desdobramentos propostos por Song et al. (2013), ao mencionar a necessidade de se desenvolver um estudo e planejamento computacional que determine a ordem de montagem das ERs geradas pela ferramenta feita pelos autores.

Os principais tópicos que sintetizam esta lacuna são:

(a) possibilidade de se converter estruturas temporárias em permanentes não é bem definida ou explorada;

(b) o processo de montagem de estruturas permanentes pode ser muito complexo; e

(c) os estágios intermediários de montagem são, muitas vezes, desconsiderados.

\section{Conclusões}

Estruturas recíprocas em madeira podem ser capazes de responder a demandas de sustentabilidade ambiental e complexidade da arquitetura contemporânea. Neste trabalho foi apresentada uma RSL que analisou 49 artigos dentre 180 resultados encontrados. É importante lembrar que apesar do estudo ter identificado muitos artigos, outros podem ter escapado a essa busca por causa de diferentes nomenclaturas ou da não indexação nas bases utilizadas. A análise desses artigos permitiu identificar e compilar lacunas que podem orientar futuros estudos envolvendo o uso dessas estruturas.

A leitura dos artigos nos permitiu ter uma melhor compreensão das origens históricas e da relevância desse tipo de estrutura, bem como das diferentes abordagens e estratégias de produção. As lacunas identificadas indicam linhas de pesquisa que poderiam contribuir para uma maior aplicabilidade das ERs. Alguns dos problemas exigem tempo para ser solucionados, como as demandas por processamento computacional e a necessidade de avanços tecnológicos em outras áreas - principalmente computação e robótica. Entretanto, novas implementações computacionais de métodos de análise estrutural e a criação de novos detalhes construtivos, que considerem os mecanismos de conexão e as propriedades do material, podem contribuir com a geração de estruturas mais interessantes e viáveis sob diferentes aspectos. 
Além disso, ao correlacionar lacunas identificadas por diferentes autores, vislumbra-se a possibilidade de solucionar aspectos de eficiência estrutural, fabricação e montagem pelo aprofundamento em estudos e pesquisas sobre as conexões, uma vez que elas podem ser responsáveis pelo tipo de transmissão de força entre os elementos, atuando como mecanismo de suporte para os estágios intermediários de montagem. Há também a oportunidade de acumular a complexidade dos sistemas nesses elementos, fazendo com que eles absorvam a transição de geometrias de leques em uma mesma estrutura.

As lacunas acima identificadas servirão como diretrizes para o desenvolvimento futuro, por estes autores, de um estudo teórico-prático sobre a produção de conexões para estruturas recíprocas em madeira, composta por elementos lineares irregulares, considerando seu comportamento estrutural e utilizando recursos de modelagem algorítmica e paramétrica e fabricação digital.

\section{Referências}

ANASTAS, Y.; RHODE-BARBARIGOS, L.; ADRIAENSSENS, S. Design-to-construction workflow for cell-based pattern reciprocal free-form structures. Journal of the International Association for Shell and Spatial Structures, v. 57, n. 2, p. 159-176, 2016.

ARAULLO, R. 3D Growth Morphology: tectonics of custom shapes in reciprocal systems. In: THE ASSOCIATION FOR COMPUTER-AIDED ARCHITECTURAL DESIGN RESEARCH IN ASIA, 23., Beijing, 2018. Proceedings [...] Hong Kong: CAADRIA, 2018.

ARAULLO, R.; HAEUSLER, M. H. Asymmetrical double-notch connection system in planar reciprocal frame structures. In: THE ASSOCIATION FOR COMPUTER-AIDED ARCHITECTURAL DESIGN RESEARCH IN ASIA, 22., Suzhou, 2017. Proceedings [...] Hong Kong: CAADRIA, 2017.

AVELLANEDA, O. F. et al. Experimental workshop in unconventional structures: deployable \& tree-like structures SMiA - training and research at Barcelona tech. In: INTERNATIONAL ASSOCIATION FOR SHELL AND SPATIAL STRUCTURES, Brasilia, 2014. Proceedings [...] Brasília: IASS, 2014.

BARRALLO, J; GONZÁLEZ-QUINTIAL, F.; SÁNCHEZ-PARANDIET, A. Laminar constructions and reciprocal structures. Nexus Network Journal: Architecture and Mathematics, v. 19, n. 3, p. 723-739, 2017.

BAVEREL, O. et al. Nexorades. International Journal of Space Structures, v. 15, n. 2, p. 155-159, 2000.

BAVEREL, O. et al. Workshop: nexorades with composites materials. International Journal of Space Structures, v. 26, n. 4, p. 363-364, 2011.

BAVEREL, O.; LARSEN, O. P. A Review of woven structures with focus on reciprocal systems: nexorades. International Journal of Space Structures, v. 26, n. 4, p. 281-288, 2011.

BAVEREL, O.; NOOSHIN, H. Nexorades based on regular polyhedra. Nexus Network Journal: Architecture and Mathematics, v. 9, n. 2, p. 281-298, 2007.

BAVEREL, O.; PUGNALE, A. Reciprocal systems based on planar elements: morphology and design explorations. Nexus Network Journal: Architecture and Mathematics, v. 16, n. 1, p. 179-189, 2014.

BERNABEU LARENA, A.; GARCÍA MÉNENDEZ, D. Extension of euskalduna conference centre and concert hall: a contemporary application of irregular reciprocal frames. Structural Engineering International, v. 24, n. 1, p. 63-67, 2014.

BROCATO, M. Reciprocal frame: kinematical determinacy and limit analysis. International Journal of Space Structures, v. 26, n. 4, p. 343-358, 2011.

DOUTHE, C.; BAVEREL, O. Design of nexorades or reciprocal frame systems with the dynamic relaxation method. Computers and Structures, v. 87, p. 1296-1307, 2009.

DRESCH, A.; LACERDA, D. P.; ANTUNES JÚNIOR, J. A. V. Design science research: a method for science and technology advancement. Suíça: Springer International Publishing, 2015.

FLOMINATOR. Disponível em: https://commons.wikimedia.org/wiki/File:Da_vinci_bridge.jpg. Acesso em: 21 jan. 2020.

GARAVAGLIA, E. et al. Collapse behavior in reciprocal frame structures. Structural Engineering and Mechanics, v. 46, n. 4, p. 533-547, 2013. 
GARCIA PUYOL, A. Mass customization of reciprocal frame structures. In: INTERNATIONAL ASSOCIATION FOR SHELL AND SPATIAL STRUCTURES, Amsterdam, 2015. Proceedings [...] Amsterdam: IASS, 2015.

GAUZIN-MÜLLER, D. Arquitectura ecológica: 29 ejemplos. São Paulo: Paralaxe, 2005.

GELEZ, S.; AUBRY, S.; VAUDEVILLE, B. Behavior of a simple nexorade or reciprocal frame system. International Journal of Space Structures, v. 26, n. 4, p. 331-342, 2011 b.

GELEZ, S.; AUBRY, S.; VAUDEVILLE, B. Nexorade or reciprocal frame system applied to the design and construction of a $850 \mathrm{~m} 2$ archaeological shelter. International Journal of Space Structures, v. 26, n. 4, p. 303-311, 2011a.

GELEZ, S.; SABY, V. Workshop: ateliers design: nexorades, facing an emergency situation. International Journal of Space Structures, v. 26, n. 4, p. 369-371, 2011.

GHEORGHE, A.; VIERLINGER, R. DigDesFab15 Research Pavillion. Frontiers in Digital Humanities, V. 4, n. 18, p. 01-07, 2017.

GHERARDINI, F.; LEALI, F. reciprocal frames in temporary structures: an aesthetical and parametric investigation. Nexus Network Journal: Architecture and Mathematics, v. 19, n. 1, p. 741-762, 2017.

GUTIERREZ, N.; FLORES, A.; PRECIADO, A. Reciprocal frame structures, a first academic approach to sustainable structures. In: INTERNATIONAL ASSOCIATION FOR SHELL AND SPATIAL STRUCTURES, Tokyo, 2016. Proceedings [...] Tokyo: IASS, 2016.

INCITE Researchers from UK. 2005. Disponível em:

https://commons.wikimedia.org/wiki/File:2005_Serpentine_Gallery_(Alvaro_Siza_and_Souto_Moura).jpg. Acesso em: 21 jan. 2020.

KITCHENHAM, B. Procedures for performing systematic reviews: technical report. New Castle \& Eveleigh (TR/SE-0401), 2004.

KOHLHAMMER, T. et al. Design and structural analysis of complex timber structures with glued T-joint connections for robotic assembly. International Journal of Space Structures, v. 32, n. 3/4, p. 199-215, 2017.

KOHLHAMMER, T.; KOTNIK, T. Systemic behaviour of plane reciprocal frame structures. Structural Engineering International, v. 21, n. 1, p. 80-86, 2011.

LARSEN, O. P. Reciprocal Frame (RF) structures: real and exploratory. Nexus Network Journal: Architecture and Mathematics, v. 16, n. 1, p. 119-134, 2014.

LARSEN, O. P. Reciprocal Frame architecture. New York: Architectural Press, 2008.

LARSEN, O. P. Reciprocal frames workshops at the royal danish academy of fine arts school of architecture in Copenhagen. International Journal of Space Structures, v. 26, n. 4, p. 365-367, 2011.

LARSEN, O. P.; CHILTON, J. C.; CHOO, B. S. Rapid construction of modular buildings using the "Reciprocal Frame". Transactions on the Built Environment, v. 21, n. 1, p. 73-82, 1996.

LARSEN, O. P.; LEE, D. S.-H. Reciprocal Frames (RFs) used for quick-built deployable emergency shelters. In: INTERNATIONAL ASSOCIATION FOR SHELL AND SPATIAL STRUCTURES, Amsterdam, 2013. Proceedings [...] Amsterdam: IASS, 2013.

MACHADO, F. A.; RUSCHEL, R. C. Soluções integrando BIM e internet das coisas no ciclo de vida da edificação: uma revisão crítica. PARC Pesquisa em Arquitetura e Construção, Campinas, v. 9, n. 3, p. 240-258, set. 2018.

MAXWELL, I. et al. Fabrication aware form-finding: a combined quasi-reciprocal timber and discontinuous post-tensioned concrete structure. In: THE ASSOCIATION FOR COMPUTER-AIDED

ARCHITECTURAL DESIGN RESEARCH IN ASIA, 34., Los Angeles, 2014. Proceedings [...] Los Angeles: ACADIA, 2014.

MESNIL, R. et al. Form finding of Nexorades using the translation method. Automation in Construction, v. 95, p. 142-154, 2018. 
NABAEI, S. S.; BAVEREL, O; WEINAND, Y. Mechanical form-finding of the timber fabric structures with dynamic relaxation method. International Journal of Space Structures, v. 28, n. 3/4, p. 197-214, 2013.

NABAEI, S. S.; WEINAND, Y. geometrical description and structural analysis of a modular timber structure. International Journal of Space Structures, v. 26, n. 4, p. 321-330, 2011.

PANTAZIS, E.; GERBER, D. Material swarm articulations: new view reciprocal frame canopy. In: EDUCATION AND RESEARCH IN COMPUTER AIDED ARCHITECTURAL DESIGN IN EUROPE, 32., New Castle, 2014. Proceedings [...] Los Angeles: eCAADe, 2014.

PARIGI, D.; KIRKEGAARD, P. H. Design and fabrication of free-form reciprocal structures. Nexus Network Journal: Architecture and Mathematics, v. 16, n. 1, p. 69-87, 2014b.

PARIGI, D.; KIRKEGAARD, P. H. The Reciprocalizer: an agile design tool for reciprocal structures. Nexus Network Journal: Architecture and Mathematics, v. 16, n. 1, p. 61-68, 2014a.

PARIGI, D.; PUGNALE, A. Three-dimensionality in reciprocal structures: concepts and generative rules. Nexus Network Journal: Architecture and Mathematics, v. 16, n. 1, p. 151-177, 2014.

PARIGI, DaD. et al. Static and kinematic formulation of planar reciprocal assemblies. Nexus Network Journal: Architecture and Mathematics, v. 16, n. 1, p. 37-59, 2014.

PUGNALE, A.; SASSONE, M. Structural reciprocity: critical overview and promising research / design issues. Nexus Network Journal: Architecture and Mathematics, v. 16, n. 1, p. 09-35, 2014.

RIZZUTO, J. P.; LARSEN, O. P. Connection systems in reciprocal frame and mutually supported elements space structure networks. International Journal of Space Structures, v. 25, n. 4, p. 243-256, 2010.

ROKKO-SHIDARE. Disponível em: https://commons.wikimedia.org/wiki/File:Rokko-shidare_六甲枝垂れ -Rokko_garden_terrace_B217107.JPG. Acesso em: 21 jan. 2020.

SAIDANI, M.; BAVEREL, O.; CROSS-RUDKIN, P. S. M. Investigation into a new type of multireciprocal grid. International Journal of Space Structures, v. 13, n. 4, p. 215-218, 1998.

SÁNCHEZ-SÁNCHEZ, J.; ESCRIG PALLÁRES, F.; RODRÍGUEZ-LEÓN, M. T. Reciprocal tree-like fractal structures. Nexus Network Journal: Architecture and Mathematics, v. 16, n. 1, p. 135-150, 2014.

SÉNÉCHAL, B.; DOUTHE, C.; BAVEREL, O. Analytical investigations on elementary nexorades. International Journal of Space Structures, v. 26, n. 4, p. 313-320, 2011.

SONG, P. et al. An interactive computational design tool for large reciprocal frame structures. Nexus Network Journal: Architecture and Mathematics, v. 16, n. 1, p. 109-118, 2014.

SONG, P. et al. Reciprocal frame structures made easy. ACM Transactions on Graphics, v. 32, n. 4, p. 94:1-94:10, 2013.

TAMKE, M.; RIIBER, J.; JUNGJOHANN, H. Generated Lamella. In: ACADIA 10: LIFE IN:FORMATION, 30., New York, 2010. Proceedings [...] New York: ACADIA, 2010.

THONNISSEN, U. A Form-finding instrument for reciprocal structures. Nexus Network Journal: Architecture and Mathematics, v. 16, n. 1, p. 89-107, 2014.

THONNISSEN, U.; WERENFELS, N. Reciprocal frames: teaching experiences. International Journal of Space Structures, v. 26, n. 4, p. 369-371, 2011.

TONG, Z.; ZHOU, R. Design and fabrication of 3D reciprocal frame structure. In: CAADRIA, 21., Melbourne, 2016. Proceedings [...] Hong Kong: CAADRIA, 2016.

\section{Agradecimentos}

Os autores agradecem o suporte garantido pela Fundação de Amparo à Pesquisa do Estado de São Paulo (Fapesp), referente ao processo n. 2019/04043-2. 


\section{Caio Magalhães Castriotto}

Faculdade de Engenharia Civil, Arquitetura e Urbanismo | Universidade Estadual de Campinas | Rua Saturnino de Brito, 224, Cidade

Universitária | Campinas - SP - Brasil | CEP 13083-889 | Tel.: (19) 3521-2426 | E-mail: caio.castriotto@gmail.com

Gabriela Celani

Faculdade de Engenharia Civil, Arquitetura e Urbanismo | Universidade Estadual de Campinas | Tel.: (19) 3521-2909 | E-mail:

celani@unicamp.br

Felipe Tavares da Silva

Departamento de Arquitetura e Urbanismo | Universidade Federal da Paraíba | Cidade Universitária, s/n, Castelo Branco | João Pessoa PB - Brasil | CEP 58051-900 | Tel.: (83) 3216-7155 | E-mail: felipe.estruturas@gmail.com

\section{Ambiente Construído}

Revista da Associação Nacional de Tecnologia do Ambiente Construído

Av. Osvaldo Aranha, $99-3^{\circ}$ andar, Centro

Porto Alegre - RS - Brasil

CEP $90035-190$

Telefone: +55 (51) 3308-4084

Fax: +55 (51) 3308-4054

www.seer.ufrgs.br/ambienteconstruido

E-mail: ambienteconstruido@ufrgs.br 